\title{
Currículo y desarrollo turístico en Guanacaste, Costa Rica: Un análisis de los niveles de educación técnico y superior
}

\author{
Curriculum and Tourism Development in Guanacaste, Costa Rica: An Analysis of the \\ Technical and Higher Education Levels
}

\section{Currículo e desenvolvimento do turismo em Guanacaste, Costa Rica: Uma análise dos níveis de ensino técnico e superior}

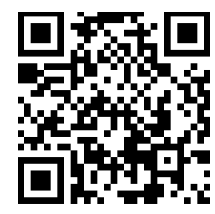

\author{
Esteban Barboza-Núñez ${ }^{1}$ \\ Universidad Nacional \\ Nicoya, Costa Rica \\ ezteban@hotmail.com
}

\begin{abstract}
Recibido • Received • Recebido: 16 / 08 / 2016
Corregido • Revised • Revisado: 24 / 11 / 2017

Aceptado • Accepted • Aprovado: 15/ 12 / 2017
\end{abstract}

\begin{abstract}
Resumen: El siguiente trabajo estudia las orientaciones curriculares de la educación técnica y superior enfocadas en turismo impartidas en colegios técnicos profesionales y universidades públicas en la provincia de Guanacaste, Costa Rica, y las contrasta con los tipos de desarrollo turístico de la región. Se analiza la relación existente entre la capacitación y la enseñanza del turismo, los contenidos curriculares a nivel técnico y superior, y las supeditaciones laborales de jóvenes de 16 a 25 en el mercado laboral al estudiar turismo. Se contrastan también los contenidos temáticos del estudio del turismo a nivel técnico y superior con las realidades y necesidades de la región como tal. Se parte de enfoques críticos de los distintos tipos de desarrollo turístico en América Latina y sus consecuencias socioculturales en las poblaciones locales. Se sugieren, al mismo tiempo, y devenidas del análisis curricular, transformaciones en la enseñanza del turismo en la provincia, de modo que el fenómeno se estudie de maneras más abarcadoras y críticas.
\end{abstract}

Palabras claves: Estudios turísticos; currículo educativo; desarrollo turístico; Guanacaste.

\footnotetext{
${ }^{1}$ Costarricense, profesor asociado de la Universidad Nacional, Mágister Litterarum por la Universidad de Costa Rica, actualmente cursa el Doctorado en Estudios de la Sociedad y la Cultura, en la misma institución. Se desempeña como docente e investigador en la Universidad Nacional desde el año 2002. Miembro fundador y proponente de la Red de Investigación en Turismo, Sociedad y Ambiente, de la Universidad Nacional; coordinador de la Cátedra Antonio Maceo, de la misma institución. Ha llevado a cabo investigaciones y ha publicado trabajos en países como Costa Rica, Estados Unidos, Cuba, México y Ecuador acerca del turismo desde una perspectiva teórica y epistemológica, los estudios postcoloniales, los estudios culturales, la literatura costarricense, y la historia local. En este último ramo, ha publicado en coautoría el libro Costa Rica en Antonio Maceo: Un legado recíproco.
} 
doi: http://dx.doi.org/10.15359/ree.22-1.20

URL: http://www.una.ac.cr/educare

CORREO: educare@una.cr

\begin{abstract}
This paper studies the curricular orientation of technical and higher education focused on tourism in technical and public universities in the province of Guanacaste, Costa Rica. It also contrasts this orientation with the types of tourism development existing in that province. In addition, the article analyzes the existing relationship between the training, the teaching of tourism, the curricular contents at a technical and higher level, and the job possibilities of young people aged 16-25 in the labor market. Moreover, the paper contrasts the contents of tourism programs at a technical and higher level with the social needs of the region. This paper is based on the critical approaches to tourism development in Latin America and the sociocultural consequences on local people. Finally, it suggests some changes in the teaching of tourism in Guanacaste so that more critical and encompassing approaches to the study of the field can become possible.
\end{abstract}

Keywords: Tourism studies; education curriculum; tourism development; Guanacaste.

Resumo: Esta pesquisa estuda as diretrizes curriculares do ensino técnico e superior focado em turismo, ministradas em colégios técnicos profissionais e universidades públicas na província de Guanacaste, Costa Rica, e contrasta com os tipos de desenvolvimento do turismo da região. O que se analisa neste trabalho é a relação entre a formação e a educação em turismo, os conteúdos do currículo em nível técnico e superior, e o trabalho supeditado jovens de 16 a 25 anos no mercado trabalhista, ao estudar turismo. Também se faz um contraste entre o conteúdo temático do estudo do turismo em nível técnico e superior com as realidades e necessidades da região como tal. A abordagem é feita a partir de enfoques críticos dos diferentes tipos de desenvolvimento turístico na América Latina e suas consequências socioculturais sobre as populações locais. São sugeridas, ao mesmo tempo, a partir da análise do currículo, mudanças no ensino do turismo na província, de modo que o fenômeno seja estudado de forma mais crítica e abrangente.

Palavras-chave: estudos turísticos, currículo educativo, desenvolvimento do turismo, Guanacaste.

\title{
Introducción
}

Durante la última década del siglo XX y en lo que llevamos del siglo XXI, la provincia de Guanacaste, localizada en la parte noroccidental de Costa Rica, ha servido como base principal para el desarrollo de polos de atracción turística alrededor del sol y la playa. En un principio ideados en 1979, con la aprobación de la Ley reguladora del Proyecto turístico Golfo de Papagayo (Picón, Parada, Baltodano, 2006), que destinó ciertas regiones de la provincia para uso exclusivo del turismo, estos polos llegarían a convertirse en la mayor fuente de atracción de inversión extranjera directa en la región, ya para el año 2007 concentra la provincia el índice de inversión inmobiliaria extranjera más alto del país, un 29\% del total (Cañada, 2011).

Se construyó un aeropuerto internacional en Liberia, la principal ciudad de la provincia, inaugurado con vuelos desde el exterior en 1995, y destinado al transporte de turistas hacia esos destinos recientemente ideados; se impulsó gran cantidad de proyectos inmobiliarios en lugares 
como el Golfo de Papagayo, El Coco, Tamarindo, o Flamingo; se construyeron hoteles cinco estrellas de la modalidad todo incluido en varios de esos parajes; se generaron desplazamientos de poblaciones, e incluso, paulatinamente, y como consecuencia del auge del mercado inmobiliario, muchos caseríos, como por ejemplo Tamarindo o El Coco, sufrieron cambios tan radicales que se tornaron irreconocibles para quien los hubiese conocido antes de la era del turismo.

En el marco de todas estas transformaciones, tanto a nivel económico, político y cultural, generados a partir del gran impulso dado a la industria turística, especialmente la desarrollada alrededor del sol y la playa y del turismo residencial -aunque en los últimos años otras variantes turísticas empiezan a darse en la región-, cabe preguntarse acerca del lugar de la juventud al respecto y, más específicamente, el papel de las políticas educativas públicas enfocadas en estos grupos etarios a la luz de tales cambios y del impulso y posicionamiento del turismo en la provincia.

Una manera de responder a esta interrogante es a través del estudio de las orientaciones curriculares en educación técnica y superior, enfocada a jóvenes de 16 a 25 años, en relación con el turismo a partir de la implantación de la industria como principal fuente de desarrollo de la provincia. Así mismo, derivándose de dicho estudio, es conveniente examinar cómo la oferta educativa responde a las particularidades de la región en cuanto a desempleo, bajos ingresos y pobreza -por ejemplo, Guanacaste tiene, después de la Región Brunca, los índices más altos de pobreza del país (Sauma, 2011)-, y en qué lugares o escalafones del complejo conglomerado turístico de Guanacaste se pretende posicionar a la juventud.

La relevancia de este análisis subyace en la necesidad de ahondar en las implicaciones de los modelos de desarrollo turístico en las opciones laborales a nivel técnico y profesional de la juventud de la región, en especial porque la actividad turística se ha posicionado, en las últimas décadas, como una importante alternativa de trabajo entre personas jóvenes en la provincia; además, ha demostrado conllevar una amplia gama de implicaciones de diversa índole, en lo social, ambiental, cultural, y laboral. Por otra parte, es importante contrastar estos modelos de desarrollo con las tendencias curriculares de las instituciones educativas de la región, no solo para analizar los planes educativos en relación con las supeditaciones laborales que el mercado pueda condicionar, sino también para estudiar en qué medida estos planes de estudio representan una respuesta a las necesidades de la zona.

\section{Desarrollo turístico en Guanacaste: Características y tendencias}

Como punto inicial, es importante llegar a un consenso en cuanto a qué entendemos por turismo y cómo vamos a usar dicho concepto en el presente trabajo, ya que de eso depende, en gran medida, el tipo de análisis de la malla curricular de la región enfocada en el ramo. En principio parece una tarea simple, ya que podríamos suponer el turismo como un servicio, y la capacitación 
de la juentud como un entrenamiento para brindar servicios en actividades turísticas. Sin embargo, tanto el turismo como el desarrollo de capacidades en jóvenes que quieren desenvolverse en dicha actividad, tanto epistemológica como curricularmente, no son cuestiones fáciles de definir.

La Organización Mundial delTurismo (Sandro, 1998) establece que el principal objetivo del turismo es "contribuir al crecimiento económico, a un desarrollo incluyente y a la sostenibilidad ambiental" (p. 46). La misma organización define la gestión turística como el conjunto de actividades que realizan las personas en lugares y estancias distintas a su entorno habitual, por un período de tiempo consecutivo inferior a un año con fines de ocio, negocios y otros; y el sistema turístico es detallado como la interacción de un conjunto de factores tales como la demanda, la oferta, el espacio geográfico, y los operadores del mercado.

La realidad, sin embargo, y al menos según se ha desarrollado el turismo en Guanacaste, parece ser mucho más compleja. La región cuenta con muchísimas más aristas que, definitivamente, hacen que esta definición, a menudo tomada como didáctica y enormemente difundida y bastante legitimada, simplemente no baste para definir y entender el fenómeno ni su modo de abordaje, en especial en torno al análisis de las orientaciones curriculares en la educación técnica y superior en turismo.

El desarrollo turístico en Guanacaste no ha escapado de la controversia y la crítica, específicamente en cuanto a su constitución, el modo en que se han desarrollado los polos turísticos, y los conflictos socioambientales generados a partir de este. Hernández y Picón (2011) señalan, por ejemplo, que "el turismo, como actividad, no ha transformado solo las actividades productivas, sino que afecta el modo de vida de las comunidades rurales, pues ha provocado cambios sociales importantes y presión sobre recursos como el agua y el espacio litoral" (p. 32). Esto se debe, precisamente, a los principales tipos de turismo que se han impulsado en la región, -el de sol y playa y el residencial- y que no necesariamente se explican a través de la definición establecida por la Organización Mundial del Turismo.

Estos tipos de turismo han generado una gran cantidad de inversión extranjera directa. Las principales inversiones se centran alrededor de hoteles de cadenas transnacionales que operan bajo el esquema "todo incluido," y en iniciativas particulares de turismo residencial y complejos inmobiliarios que incluyen campos de golf, marinas, servicios de salud, y centros comerciales y de entretenimiento (Cañada, 2011). Sin embargo, uno de los problemas que pueden derivar de la concentración de la inversión y el desarrollo en este tipo de turismo, según Aledo (2008), es que llega a constituir una unidad de ocio privatizada, autónoma e independiente del territorio en el que se instala, es decir, un tipo de burbuja turística o enclave.

A pesar de existir, en algunas regiones de la provincia, especialmente en las partes altas, alrededor de volcanes como el Rincón de la Vieja, el Miravalles o alrededor del lago Arenal, una 
oferta turística que difiere sustantivamente de la que predomina en el litoral, lo cierto es que tanto en inversión, infraestructura e impacto, el turismo de sol y playa y el turismo residencial se yerguen como las principales cartas de presentación de Guanacaste para atraer tanto turistas como capital extranjero, y de ninguna manera son factores a ignorar a la hora de abarcar el estudio del fenómeno turístico de la provincia.

Ahora bien, ¿qué está haciendo el sistema educativo formal público, tanto técnico como superior, al respecto? ¿Cómo está respondiendo al fenómeno, con los claroscuros que este presenta? ¿Cuáles orientaciones curriculares predominan en la oferta académica? ¿Se amolda el sistema a las demandas del mercado, o propone alternativas al modelo? Es en este punto donde se da la intersección entre el sistema educativo enfocado al turismo y la industria turística en sí, y en donde se pueden estudiar las orientaciones curriculares a la luz de las especificidades de la industria.

\section{Algunas corrientes en la didáctica del turismo en la educación técnica y superior}

Los programas de educación turística, vistos desde la perspectiva curricular, entendiendo el currículo como las prácticas educativas que proveen experiencias que posibilitan la continua reconstrucción de estas que añade a su significado y que aumenta la habilidad individual para dirigir el curso de experiencias subsecuentes (Hyun, 2006), a nivel superior tienden a subrayar las tensiones y las divisiones entre esta área, considerada interdisciplinaria. Según Hall y Brown (2008), a nivel universitario algunos cursos enfatizan la evaluación crítica del contexto y las consecuencias del turismo y los procesos de desarrollo, a través de la óptica de análisis de las políticas de planificación, ecología y estudios etnográficos.

A menudo, también se intenta integrar los ámbitos empíricos y conceptuales del quehacer académico para colocar el desarrollo turístico dentro del marco de evaluación de procesos de grandes cambios. Es decir, existe la inclinación a abordar el turismo desde diferentes perspectivas analíticas que van más allá de la simple capacitación de jóvenes para cumplir una función específica en el ámbito empresarial. Estos enfoques tratan de superar la idea de la actividad como una simple transacción en la que al estudiantado se le capacita para dar un servicio específico que satisfaga las demandas y las necesidades de los grupos de turistas.

En cuanto a producción intelectual, desde finales del siglo pasado hasta la actualidad, esta ha aumentado en América Latina, especialmente en países como Brasil y México, entre otros, y ha influido enormemente en el diseño curricular de las carreras de turismo, tornándolas más críticas y abarcadoras, haciendo del turismo un campo de estudio, más que un servicio. La mayoría de publicaciones advierten que "la investigación y la producción del conocimiento en turismo requiere de un tipo de actitud que se caracterice por ser enjuiciativa, crítica, interpretativa y transformadora de la sociedad en general" (Castillo y Panosso, 2010, p. 31). Incluso, a nivel 
epistemológico, existen debates enriquecedores acerca de si es o no el turismo una ciencia, o si está en proceso de serlo, o si tan solo es una actividad humana, y qué implicaciones tiene cada una de estas tendencias (Lohmann y Panosso, 2012). Estas posiciones complejizan y enriquecen el campo como área de estudio y de trabajo mucho más allá de un servicio o una transacción.

Tan solo la existencia de estos debates y estos cuestionamientos permite potenciar el campo de estudio del turismo en institutos y universidades, y las áreas consideradas como servicios se convierten en escenarios que involucran distintos saberes que se ocuparán ya no solamente de la enseñanza de la facilitación de un servicio, sino de crear toda una dialéctica en cuanto a la naturaleza del servicio en sí. De este modo, una escuela de gastronomía, por ejemplo, se convierte en un centro de estudio de los procesos culturales, históricos y sociales de la comida de una región en particular, se escruta su valor e importancia cultural entre las comunidades locales, su importancia nutritiva, y deja de ser un lugar donde simplemente se enseña a elaborar comidas o bebidas para un restaurante u hotel. Lo mismo se puede decir de otras particularidades de turismo, como el alojamiento y el impacto ambiental, la relación entre la actividad y la cultura, la economía y las identidades de una región o país.

Por otro lado, en programas más básicos, como por ejemplo en educación técnica, se enfatiza la parte práctica, los negocios, el entrenamiento vocacional, y la capacitación para trabajar en las realidades que conforman las actividades turísticas y el sector de viajes. En este nivel, el papel de la ética, la responsabilidad y la evaluación crítica es menos evidente (Hall y Brown, 2008). Se enfatiza la enseñanza de servicios básicos de hotelería, alimentos, bebidas, o atención al cliente de modo que se satisfagan las demandas y necesidades de un público turista consumidor de un servicio según las normativas del mercado.

Esto hace surgir interrogantes en cuanto a si las capacitaciones en educación técnica simplemente preparan estudiantes para satisfacer las demandas del medio, en este caso, el que mayoritariamente se impone en Guanacaste, o si existen posibilidades, dentro de esta misma, de enfoques que apunten hacia una diversificación de la oferta, y a tipos de turismo más sustentables, como por ejemplo, el turismo rural, o el turismo ecológico, o bien, permitan desarrollar el emprendedurismo entre el estudiantado. La comparación entre estas tendencias curriculares y las de los programas de enseñanza del turismo tal y como se implementan en Guanacaste es lo que nos permitirá conocer mejor sus orientaciones curriculares y su pertinencia en la actividad turística tal y como esta se ha desarrollado en la zona.

\section{Capacitación técnica y superior alrededor del turismo en Guanacaste: Tendencias y posibilidades}

Para el estudio de las orientaciones curriculares del turismo, el presente trabajo se circunscribirá a la educación técnica, impartida porla figura de los colegios técnicos profesionales.

6 Esteban Barboza-Núñez

Los artículos de la Revista Electrónica Educare del Centro de Investigación y Docencia en Educación de la Universidad Nacional, Costa Rica, se comparten bajo términos de la Licencia Creative Commons: Reconocimiento, № Comercial, Sin Obra Derivada 3.0 Costa Rica. Las autorizaciones adicionales a las aquí delimitadas se pueden obtener en el correo: educare@una.cr 
Un total 14 colegios de este tipo imparten especialidades en turismo en la provincia, y son dependientes de los programas del Ministerio de Educación Pública. Las universidades públicas que imparten la carrera en la región son la Universidad de Costa Rica, en la Sede Guanacaste, en el Campus de Liberia y la Universidad Nacional, Sede Regional Chorotega, en los Campus Liberia y Nicoya.

En ninguno de estos dos sectores existen programas diferenciados y diseñados específicamente para Guanacaste, aunque las carreras de turismo en la Universidad Nacional y en la Universidad de Costa Rica sí nacieron en la provincia, y no emanan de otras sedes de la universidad. En la Universidad de Costa Rica se imparte desde 1991, y en la Universidad Nacional desde el 2001 (Arrieta y Rivera, 2007). Otra institución importante que imparte materias relacionadas con el turismo, específicamente a nivel técnico, es el Instituto Nacional de Aprendizaje, pero para este trabajo ha sido excluido, ya que no existen rangos etarios específicos entre los interesados en estudiar estas materias, como sí sucede en los colegios técnicos y en las universidades, por lo tanto, el análisis se centrará en la educación técnica del Ministerio de Educación Pública y las universidades.

\section{El caso de la educación técnica}

Según datos proporcionados por la Memoria institucional 2006-2014 del Ministerio de Educación Pública (s. f.), la educación técnica que se brinda en Costa Rica ha pasado por un proceso de modernización que responde al hecho de que dos terceras partes de la población labora en el sector de servicios, y en el hecho de que todos los sectores productivos han sufrido procesos de modernización. Esto deja ver la importancia que se le da a la parte laboral y a la colocación de estudiantado graduado en puestos de trabajo, y la estrecha relación que existe con el sector productivo. Dentro de las especialidades brindadas hay siete agropecuarias, 24 industriales y 24 de comercio y servicios, entre los que se ubica el turismo. La importancia dada a la parte industrial y a la parte de comercio y servicios, al tener mayor oferta, deja ver lo amoldados que están dichos centros educativos a los cambios productivos que ha experimentado el país desde mediados del siglo XX.

Específicamente en cuanto al área de turismo, las especialidades son Turismo en Alimentos y Bebidas, Turismo Ecológico, Turismo en Hotelería y Eventos Especiales, Turismo Rural, y Turismo Costero. En el caso de Guanacaste, según datos consultados directamente en los colegios técnicos profesionales de la región, todas las especialidades, menos el turismo costero, se imparten en la provincia, como se aprecia en el siguiente cuadro, elaborado a partir de consultas directas a los centros educativos. 
doi: http://dx.doi.org/10.15359/ree.22-1.20

URL: http://www.una.ac.cr/educare

CORREO: educare@una.cr

Tabla 1: Colegios técnicos que imparten turismo y número de estudiantes

\begin{tabular}{|c|c|c|c|}
\hline CTP & Cantón & Especialidades & Estudiantes \\
\hline Liberia & Liberia & Eventos especiales / Alimentos y bebidas & 10 \\
\hline Fortuna & Bagaces & Turismo ecológico/ Alimentos y bebidas & 16 \\
\hline Nicoya & Nicoya & Eventos especiales & 30 \\
\hline La Mansión & Nicoya & & 53 \\
\hline Copal & Nicoya & Turismo rural & \\
\hline Corralillo & Nicoya & & 45 \\
\hline Hojancha & Hojancha & Turismo ecológico / Eventos especiales & 95 \\
\hline Nandayure & Nandayure & Alimentos y bebidas / Turismo ecológico & 67 \\
\hline 27 de abril & Santa Cruz & Eventos especiales / Alimentos y bebidas & 49 \\
\hline Cartagena & Santa Cruz & Alimentos y bebidas / Eventos especiales & 96 \\
\hline Carrillo & Carrillo & & \\
\hline Sardinal & Carrillo & Alimentos y bebidas / Eventos especiales & 103 \\
\hline Santa Cruz & Santa Cruz & & \\
\hline Abangares & Abangares & Turismo ecológico / Turismo rural & 58 \\
\hline Tronadora & Tilarán & Turismo rural / Hotelería y eventos especiales & 18 \\
\hline
\end{tabular}

Nota: Elaboración propia a partir de consultas telefónicas a colegio técnicos.

El Ministerio de Educación apunta que la especialidad en turismo se encuentra influenciada por factores vinculados a la política económica nacional y global, a los cambios tecnológicos constantes, y al desarrollo tecnológico, lo que hace evolucionar los contenidos y conocimientos por impartir (Campos, 2009b). Existe un interés de la educación técnica en preparar a la juventud para que se inserte en el mundo laboral, amoldándose a los devenires del mercado y a los "nuevos modelos de globalización económica" (Campos, 2009a, b, p. 16). Ello coincide con lo apuntado por Hall y Brown (2008) anteriormente sobre la educación técnica en turismo y su concentración en capacitar para trabajar en las realidades que conforman las actividades turísticas y el sector de viajes, al mismo tiempo que se diferencia del estudio de la actividad turística desde la educación superior, generalmente más crítica e interdisciplinaria. 
Los perfiles profesionales de todo el estudiantado que optan por turismo son los mismos, independientemente de la especialidad. Los perfiles especifican funciones como interpretar información, transmitir instrucciones, dirigir procesos, proponer soluciones, utilizar herramientas tecnológicas, y demostrar ética profesional, entre otras (Campos, 2009a). Es un perfil bastante general y que no especifica mucho en relación con lo que se pretende desarrollar, independientemente de otras especialidades que, aunque muy distintas, describen perfiles muy similares.

Donde sí se puede notar los factores más específicos es en los perfiles ocupacionales, es decir, lo que se pretende que hagan una vez que se inserten en el mercado laboral. El turismo en alimentos y bebidas prepara a los técnicos para realizar inventarios, distribuir pedidos de alimentos, montar mesas, acondicionamiento de mobiliario, aplicar normas de salud e higiene, ofrecer una oferta gastronómica según el evento, y aplicar técnicas de mercadeo en la venta de servicios gastronómicos (Campos, 2009b). En el turismo ecológico se prepara al estudiantado para la conducción de grupos, la aplicación de técnicas de animación, el diseño de paquetes turísticos de educación ambiental, la utilización de programas de reservación, y la interpretación de elementos geográficos (Rodríguez y Cortés, 2001). La especialidad en hotelería enfatiza en la planificación de labores de recepción, la resolución de problemas de la clientela, elaboración de reportes de cierre de turno, organización de procedimientos operativos de restaurantes y el arreglo de habitaciones, entre otras (Campos, 2009a). Y, finalmente, el turismo rural enfatiza la administración de pequeñas y medianas empresas, venta de productos turísticos rurales, conocimientos contables para pequeñas empresas, gestión de proyectos productivos rurales de aprovechamiento turístico, interpretación del medio agroecológico e interpretación de flora y fauna, entre otros (Campos, 2010).

Se busca lograr estos perfiles ocupacionales según estructuras curriculares establecidas en los programas del Ministerio, y en los que se indican las materias, el año y el número de horas por materia. En el caso de Turismo con énfasis en alimentos y bebidas, las materias impartidas son Empresa turística, Introducción turística, Control interno y limpieza de cocina, Cocina básica, Bar y restaurante, Cocina fusión, Inglés conversacional, y Cocina internacional (Campos 2009b). En este énfasis, tanto el perfil ocupacional como la estructura curricular apuntan a una capacitación de la juventud para tareas de índole asistencial en empresas hoteleras de tamaños medianos o grandes. Se enfocan en brindar servicio al cliente y en brindar asistencia a puestos medios y altos en empresas turísticas, y a satisfacer las demandas de estos de manera puntual, según los estándares de clasificación hotelera, salud ocupacional e higiene vigentes.

En cuanto a Turismo ecológico, el programa estipula la enseñanza de materias como Recursos turísticos, Ecología, Educación ambiental, Gestión ambiental, Gestión empresarial, Inglés conversacional, Biodiversidad, y Conducción de grupos (Campos, 2010). Esta especialidad, según se desprende de la malla curricular, se centra en el manejo de grupos, la animación y la interpretación del medio ambiente. Se espera que adquieran conocimientos de guía turístico, 
aunque eso claramente queda supeditado al dominio de idiomas extranjeros, principalmente el inglés, y a la obtención de una licencia de guía, aspecto que no está contemplado en su formación. Existe un mayor contacto con los grupos de turistas y el hecho de que se le dé énfasis a la educación ambiental y a la ecología supone un mayor grado de criticidad y conciencia ambiental que el énfasis anterior.

En el caso de Turismo con énfasis en hotelería y eventos especiales, las materias abarcan Empresa turística, Introducción turística, Gestión hotelera, Control interno y limpieza de cocina, Bar y restaurante, y Cocina básica (Campos, 2009a). En este caso el programa, al igual que el de alimentos y bebidas, también apunta a un entrenamiento para tareas de índole asistencial y de manejo de sectores básicos dentro de un hotel, como la limpieza, o el área de restaurante, lo que condiciona a la juventud a operar en sectores muy específicos de un hotel, y siempre como apoyo a mandos medios y superiores.

Finalmente, la opción de Turismo rural incluye las materias de Empresa turística, Introducción turística, Control interno y limpieza de cocina, Agroturismo, Gestión de alimentos y bebidas, y Organización de grupos (Campos, 2009a). Esta opción es la única que tiende a darle más énfasis al emprendedurismo y a la explotación de recursos turísticos más allá de la oferta del sol y la playa, y sus requerimientos técnicos. Esto se nota en el énfasis en la gestión de la pequeña empresa y en el aprovechamiento de recursos turísticos rurales, según el programa.

Vemos, entonces, que la oferta técnica se orienta según la fisonomía del medio, y la prioridad es darle a la juventud una herramienta para insertarse en el mercado laboral tal y como este está constituido. A pesar de enfatizar los perfiles profesionales de los programas valores como el amor por el país, el cuidado de las tradiciones y las costumbres, el rescate de las tradiciones autóctonas, y la protección al medio ambiente (Campos, 2009a), los contenidos curriculares se enfocan en la capacitación práctica y en la capacidad de inserción en el mercado laboral a través del aprendizaje de funciones concretas, donde la evaluación crítica del fenómeno del turismo y la propuesta de alternativas es menos evidente.

\section{El caso de las universidades públicas: la Universidad de Costa Rica y la Universidad Nacional}

Como se apuntó anteriormente, en la provincia la carrera de turismo se imparte tanto en la Universidad de Costa Rica como en la Universidad Nacional. Se enseña en tres campus, el de Nicoya y el de Liberia por parte de la Sede Regional Chorotega de la Universidad Nacional, y el de Liberia en la Sede Guanacaste de la Universidad de Costa Rica. Ninguna de las dos carreras es desconcentrada, es decir, ambas nacieron en las sedes regionales, y tanto desde 1991, por parte de la Universidad de Costa Rica, como desde el 2001 por parte de la Universidad Nacional, se crearon en la provincia. 10

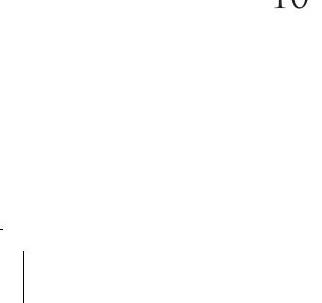


En la Universidad de Costa Rica se imparte Turismo ecológico con grado de bachillerato universitario. Su apertura en 1991 responde a un modelo de desarrollo basado en la captación de inversión extranjera, en el que se ve al turismo como una opción para sacar al país de la recesión provocada por la caída de los precios del café y el banano, y en el que se establece la necesidad de preparar profesionales en el área a nivel superior, ya que anterior a la fecha, solo el Instituto Nacional de Aprendizaje impartía turismo, a nivel técnico (Arrieta y Rivera, 2007). Su objetivo fundamental es formar profesionales comprometidos con la conservación natural, con el respeto cultural y con el desarrollo sostenible; capaces de educar y propiciar el cambio de actitudes hacia el respeto por la naturaleza y por una verdadera calidad de vida (Universidad de Costa Rica, s. f.a). Cabe resaltar también que la carrera fue inaugurada en una época en la que la ecología y la preocupación por el ambiente empezaban a cobrar relevancia -la Cumbre de Río se celebraría en 1992- y el país se promocionaba como un destino ecológico y el ecoturismo era la principal apuesta del país. De hecho, para mediados de la década de los años noventa, el slogan con que se promocionaba al país en el exterior era "Costa Rica: No Artificial Ingredients" (Segura, 1998 , p. 5), poniendo énfasis en las bondades naturales del país como lugar de atracción.

En la descripción de la carrera se dice que es una opción académica cuya finalidad es contribuir a un modelo de desarrollo sostenible, que vele por la conservación de la naturaleza, el bienestar de las comunidades y una economía sólida y equitativa (UCRa, s. f.). En cuanto al perfil profesional, se busca que el estudiantado conozca los principios del ecodesarrollo como factor de cambio en las estructuras socioeconómicas del país, organice la actividad turística en relación con los recursos naturales, sociales y económicos para favorecer el desarrollo local, valore e identifique las especies de animales y vegetación del país, se comunique correctamente en inglés, aplique principios de comunicación humana, y atienda principios jurídicos que rigen la actividad equitativa (UCR, s. f.a). Es decir, se da una combinación entre el manejo de la historia natural del país, y factores de ciencias sociales relacionados con el desarrollo y la economía, al mismo tiempo que se les capacita para el manejo de grupos de visitantes.

En cuanto al plan de estudios, aparte de las humanidades, los repertorios y los seminarios de realidad nacional, obligatorios para todo estudiante que ingresa a la universidad, existe una combinación entre materias de ciencias naturales, ciencias exactas y ciencias sociales. Hay un fuerte componente de las primeras que van desde biología, biogeografía, biosistemática, historia natural, educación ambiental, ecología, ecodesarrollo y ecoturismo. Estas materias constituyen un $35 \%$ del total de los créditos, y son el componente más fuerte de la carrera. El área de las ciencias exactas presenta cursos de matemática, cálculo y estadística. Finalmente, en cuanto a ciencias sociales, hay cursos de relaciones interpersonales, teorías de personalidad, antropología, historia, arqueología, economía y administración, y constituyen un $28 \%$ del total de créditos, es decir, el segundo componente más fuerte (UCR, s. f.b). Se nota la combinación de materias que tienen que ver con historia natural y biología con materias que tienen que ver con la relación con 
la clientela, manejo de grupos y elementos básicos de antropología. Se espera, entonces, que el estudiantado sepa interpretar la naturaleza y contribuir a procesos de desarrollo sostenible dentro de la industria turística y, al mismo tiempo, tener capacidad de trabajar con grupos e interpretarles a estos los aspectos ecológicos del turismo del país. Los cursos que más especifican algún tipo de relación con el turismo en sí son los de ecodesarrollo y ecoturismo.

Según las opciones laborales que aparecen en el descriptor de la carrera, el estudiantado graduado puede desempeñarse laboralmente en cámaras de turismo, agencias de turismo, áreas protegidas; como administrador o administradora de hoteles; en asesorías a proyectos ecoturísticos; en organizaciones no gubernamentales o estatales de múltiples perfiles; y en consultorías sobre interpretación ambiental, capacidad de carga turística, y gestión sostenible (UCR, s. f.a). Sin embargo, al menos a nivel curricular, no existe realmente una conexión entre los contenidos de los cursos y algunas funciones como las que se describen en el plan, especialmente en áreas como la administración hotelera, las agencias de viajes o las cámaras de turismo, dada la ausencia de un énfasis en cuestiones como administración hotelera o manejo de agencias de viajes.

En la Sede Regional Chorotega de la Universidad Nacional la carrera relacionada con el turismo tiene el nombre de Gestión empresarial del turismo sostenible y se imparte tanto en Liberia como en Nicoya, en este último campus desde el año 2003. La descripción de dice que proporciona conocimientos teóricos y prácticos fundamentales en la evolución de la actividad turística y ofrece los elementos básicos para valorar la recreación como un derecho humano. El plan de estudios incluye énfasis que tienen que ver con las principales actividades que se generan dentro del turismo, especialmente dentro de empresas que actúan como intermediarias entre el sujeto turista y el sujeto receptor (Universidad Nacional, s. f.b). Dentro del plan de estudios se nota un gran énfasis en cursos de administración, y una menor cantidad en sistemas turísticos, y aún menos en cuanto a patrimonio histórico y cultural.

Los cursos de administración constituyen un $42 \%$ del total de créditos e incluyen contabilidad, administración de empresas turísticas, mercadeo, finanzas, planificación, recursos humanos, y gestión de empresas gastronómicas, de hospedaje y de agencias de viajes. Los cursos relacionados con turismo como campo de estudio se circunscriben a investigación para turismo, organización comunal, geografía turística y turismo alternativo. Constituyen un $23 \%$ del total de créditos. Existe un solo curso de patrimonio cultural Bachillerato en Gestión empresarial del turismo sostenible (UNA, s. f.a). La cantidad de cursos de administración de la carrera demuestra el énfasis en la parte técnica de los procesos administrativos de empresas hoteleras de pequeño, mediano y gran tamaño, aunque también pueden dotar al estudiantado de herramientas para ciertos tipos de emprendedurismo, especialmente el establecimiento de pequeñas empresas. 
El estudiantado graduado está capacitado, según indica el descriptor del programa, para gestionar y dirigir proyectos turísticos, hacer proyecciones sobre variables que constituyen un modelo turístico de desarrollo sostenible, organizar agencias de viaje, evaluar la estructura organizacional hotelera, y diseñar, planificar, organizar, desarrollar y administrar actividades en empresas y organizaciones turísticas (UNA, s. f.b). Se nota una mayor conexión entre lo que dicta el currículo y lo que se indica que es capaz de hacer el estudiantado una vez graduado, especialmente en cuanto a la posibilidad de acceder a mandos medios en empresas turísticas y a emprender y asesorar pequeños negocios.

Si hacemos un análisis de los currículos de ambas instituciones, y los contrastamos con las características del turismo que se da en la zona expuestas anteriormente, y a las tendencias internacionales del turismo como campo de estudio e investigación, vemos que falta una mayor relación entre las universidades y sus programas, y las realidades de la región, específicamente el modo en que el desarrollo turístico ha sido implementado. En cuanto a la Universidad de Costa Rica, hay que acotar que el turismo ecológico perteneció a una tendencia en boga principalmente en la década de los años 1990, cuando se instauró la carrera, y que en su momento constituyó la mayor carta de presentación y explotación turística del país y se veía como el mejor mecanismo para disfrutar y, al mismo tiempo, conservar la naturaleza.

Existen, sin embargo, otros tipos de turismo que se han desarrollado posteriormente, especialmente a principios del siglo XXI, y que incluso han cuestionado al ecoturismo por precisamente mercantilizar la naturaleza, crear estrés ecológico y regirse por la dinámica de la acumulación de capital sin cuestionar los sistemas de poder y la inequidad, mientras se usa el concepto de turismo ecológico como una estrategia de mercadeo que encubre a la vez sus aspectos negativos (Horton, 2009). Además, también se ha cuestionado la tipología del ecoturismo por eclipsar, bajo su etimología y lo que supuestamente conlleva -apreciación y cuidado de la naturaleza y grupos turistas educados, respetuosos y responsables- otros tipos de turismo que podrían contribuir de una mejor manera al desarrollo local integral, como el turismo rural o el turismo rural comunitario (Russell y Wallace, 2004). Se podrían citar una gran cantidad de estudios críticos realizados en otras latitudes en los últimos diez años acerca del ecoturismo en América Latina, Asia y África, pero el punto es que a lo largo de 25 años, desde el diseño curricular y la creación de la carrera, ha habido un enorme aporte teórico y cuantitativo que sustenta la idea de que el ecoturismo como concepto y práctica ha sido cuestionado y ha evolucionado, algo que no se refleja en un currículo sin cambios en un cuarto de siglo, específicamente desde 1991.

No se trata de afirmar que el ecoturismo como tal está superado, ya que no es del todo cierta esta suposición, pero sí que la realidad nacional y regional ha cambiado enormemente desde 1991 y Guanacaste no se ha decantado, especialmente a partir del nuevo milenio, ni promocionalmente ni en la práctica, por el turismo ecológico. Tampoco se trata de afirmar que el currículo tenga que amoldarse a los devenires del mercado actual, pero se nota que no 
doi: http://dx.doi.org/10.15359/ree.22-1.20

URL: http://www.una.ac.cr/educare

CORREO: educare@una.cr

le presta gran atención a la problemática y a las oportunidades generadas por este, ni a sus efectos en la sociedad guanacasteca. La criticidad, interdisciplinariedad y transdisciplinariedad que han adquirido los estudios turísticos a nivel superior en redes de investigación, institutos y universidades en otras latitudes aún no se notan en un currículo estático y monista.

En cuanto a la Universidad Nacional, se nota el gran énfasis en aspectos técnicos de administración de empresas, supuestamente con énfasis en empresas turísticas, y en un menor grado, al estudio básico de sistemas turísticos, y la ausencia, con excepción de un curso de patrimonio cultural, de cursos que aborden, de manera crítica, las características del turismo y su impacto en la región.

Cuando se habla de sistemas turísticos, se tiende a abordar definiciones estandarizadas como la de la Organización Mundial de Turismo, que son más canónicasy aceptadas precisamente por su supuesta neutralidad y por no profundizar en los claroscuros de la industria. Por otra parte, en los cursos de administración se nota su generalidad -bien podrían parecer y a menudo se equiparan con cursos de la carrera de administración de empresas- y no su especialización para abordar la cuestión del turismo. No existen cursos inter y transdisciplinarios que cuestionen la problemática, y propongan soluciones o vislumbren ventajas. Se sigue viendo el estudio del turismo como una capacitación para brindar un servicio, en este caso, desde puestos medios, y no como un campo de estudios en el que varias disciplinas pueden converger e ir más allá de los aspectos técnicos y prácticos. Esto hace que la carrera en dicha universidad se parezca a la educación técnica, solamente que con más cursos y con un mayor grado académico al salir.

\section{Conclusiones}

Según Tilly (2000), las desigualdades son creadas por el imaginario social que, por medio de construcciones, condiciona a los diferentes grupos humanos a cumplir ciertas funciones en una sociedad determinada, y a través de vínculos y relaciones, junto con la explotación del trabajo, los circunscribe dentro de ciertas categorías y labores específicas. En el caso de la juventud que estudia turismo en la provincia de Guanacaste, parece ser que las orientaciones curriculares de los programas en cuestión, tanto a nivel técnico como superior, los circunscribe a un contexto laboral y académico que no le presta suficiente atención al estudio y análisis exhaustivo y enjuiciador del fenómeno, con todos los matices que este presenta en la provincia.

A nivel técnico, y como era de esperar, la parte crítica y analítica parece estar más ausente en los programas. Esto concuerda con las características del estudio del turismo a nivel técnico apuntadas anteriormente, ya que lo que se espera es que la juventud aprenda a realizar sistemáticamente ciertas labores que les permita obtener herramientas con las que se puedan insertar al mercado laboral tal y como este se presenta, de ahí lo específico de los cursos y las tareas que aprenden y que se espera que puedan desempeñar. 
Donde sí se nota que existe una dislocación entre lo que el currículo muestra, lo que se espera del perfil de salida, y las necesidades de profundizar y diversificar el estudio de la industria turística desde la interdisciplinariedad y la criticidad es en los programas universitarios. Un programa que en 25 años no muestra cambios o actualizaciones, a pesar de las profundas transformaciones en la fisonomía turística de la región, o que no ha evolucionado al lado de los estudios turísticos que, incluso, han llegado a cuestionar la naturaleza misma del tipo de turismo que se imparte, definitivamente provoca una reducción de oportunidades en la juventud que está en el programa y, más aún, condiciona a un rezago académico los estudios turísticos que puedan surgir de la universidad, en este caso la Universidad de Costa Rica.

En cuanto a la Universidad Nacional, se llega a transformar la carrera, según el currículo, casi en una especialidad en turismo de la rama de la administración de empresas. El currículo sí ha tenido cambios desde su inauguración, pero estos han ido cada vez más inclinándose hacia la parte técnico-administrativa, dejando de lado materias más teóricas y de corte sociológico y cultural. De hecho, en el año 2008 la carrera pasó de llamarse Bachillerato en Turismo a su nombre actual, incorporando en la malla más materias de administración y usando el nombre de "turismo sostenible" para supuestamente usar ese conocimiento en aras de incentivar prácticas sustentables, aunque en la realidad solo existe un curso de organización comunal en toda la carrera y ninguno que tenga que ver con sustentabilidad.

Las características del turismo tal y como este se ha ido desarrollando en Guanacaste en las últimas décadas hacen imprescindible un reacomodamiento de la oferta curricular, especialmente a nivel superior. Evidentemente no se trata de decir que la oferta se tenga que acomodar al mercado. Más bien esta debe acomodarse a las necesidades de la región más allá de lo que dicte el mercado, y debe ser un elemento sustantivo en la generación de oportunidades y equidad entre las poblaciones locales, que se han ido acostumbrando a ver el turismo como un negocio ajeno en el que solo pueden participar como sector empleado en labores específicas, mas no a tener acceso al ocio o a emprender sus propios negocios turísticos.

También deberían crearse, desde las universidades, oportunidades para investigar y estar al nivel de otras instituciones en otras latitudes en cuanto a investigación y proyectos de extensión. Al revisar índices de revistas especializadas en turismo, como la prestigiosa "Annals of Tourism Research," se ve el nivel y los rumbos que ha tomado la investigación mundial, y cuánto falta para llegar, desde las universidades nuestras a esos niveles.

Las orientaciones curriculares definitivamente condicionan el desempeño social de la juventud que estudia turismo en Guanacaste. La circunscribe a ciertos espacios y al mismo tiempo le limita el accionar dentro o fuera de estos. El abordaje crítico y constructivo del desarrollo turístico en la región adolece de propuestas analíticas y propositivas que se deriven de las mallas curriculares que integran la educación superior y técnica. Es importante integrar, especialmente desde las universidades, enfoques que permitan un estudio interdisciplinario de los procesos culturales, ambientales, territoriales y sociales devenidos de la industria, así como las transformaciones que 
esta ha generado en la fisonomía de la provincia, y que vayan mucho más allá de la interpretación del patrimonio natural o la administración de empresas turísticas. De este modo se ampliará el horizonte de expectativas de la juventud, sus opciones laborales y, sobre todo, su capacidad para contribuir al mejoramiento social, a la creación de opciones de desempeño laboral, y a hacer del turismo una práctica menos excluyente en la región y en el resto del país.

\section{Referencias}

Aledo, A. (2008). De la tierra al suelo: La transformación del paisaje y el nuevo turismo residencial. Arbor: Ciencia, Pensamiento y Cultura. 184(729). 99-113. doi: https://doi.org/10.3989/ arbor.2008.i729.164

Arrieta, G. y Rivera, G. (2007). La carrera de turismo ecológico en la Sede Guanacaste y el desarrollo del ecoturismo en la Universidad de Costa Rica. Inter Sedes, 7(13) 41-53. Recuperado de https://revistas.ucr.ac.cr/index.php/intersedes/article/viewFile/918/979

Campos, M. V. (2009a). Ministerio de Educación Pública. Programas de estudio. Programa de hotelería y eventos especiales. Recuperado de http://www.mep.go.cr/sites/default/files/ descargas/programas-de-estudio/turismohoteleriaeventos10.pdf

Campos, M. V. (2009b). Ministerio de Educación Pública. Programas de estudio. Programa de turismo en alimentos y bebidas. Recuperado de http://www.mep.go.cr/sites/default/files/ descargas/programas-de-estudio/turismoalimentosbebidas10.pdf

Campos, M. V. (2010). Ministerio de Educación Pública. Programas de estudio. Turismo Rural. Recuperado de http://www.mep.go.cr/programa-estudio/turismo-rural-0

Cañada, E. (2011). Conflictividad turística en Centroamérica. En M. Blàzquez y E. Cañada (Eds.), Nueva colonización turística: Del Mediterráneo a Mesoamérica y El Caribe. Lógicas espaciales del capital turístico (pp. 163-208). Managua: EDISA.

Castillo, M. y Panosso, A. (2010). Implicaciones epistemológicas en la construcción del conocimiento del turismo. En M. Castillo, A. Panosso (Orgs.), Epistemología del turismo: Estudios críticos (pp. 15-40). México: Trillas.

Hall, D. y Brown, F. (2008). Finding a way forward: An agenda for research. Third World Quarterly, 29(5), 1021-1032. doi: https://doi.org/10.1080/01436590802106254

Hernández, A. y Picón, J. C. (2011). En la frontera del conflict socioambiental: El modo de vida rural y el desarrollo del turismo de sol y playa en Guanacaste, Costa Rica. Ambientales, 42(2), 31-44. doi: https://doi.org/10.15359/rca.42-2.3

Horton,L.R.(2009).Buying upnature:Economicand socialimpactsofCostaRica'secotourismboom. Latin American Perspectives, 36(3), 93-107.doi: https://doi.org/10.1177/0094582X09334299 
Hyun, E. (2006). Chapter two: Curriculum understanding: Its relationship to teaching. Counterpoints, 297, 17-32.

Lohmann, G. y Panosso, A. (2012). Teoria do turismo. Conceitos, modelos e sistemas. Sao Paulo: Aleph.

Ministerio de Educación Pública. (s. f.). Memoria institucional 2006-2014. La educación subversiva: Atreverse a construir el país que queremos. San José, Costa Rica: Autor. Recuperado de http:// www.mep.go.cr/sites/default/files/page/adjuntos/memoriainstitucional20062014pdf.pdf

Picón, J., Parada, A. y Baltodano, J. (2006). La inserción de la microempresa en el conglomerado empresarial turístico: Caso Papagayo en Costa Rica. Nicoya, Costa Rica: CEMEDE.

Rodríguez, S. y Cortés, Á. (2001). Programa de estudio de la educación diversificada técnica. Especialidad Turismo Ecológico, Ministerio de Educación Pública. Recuperado de http://www. mep.go.cr/sites/default/files/descargas/programas-de-estudio/turismoecologico12.pdf

Russell, A. y Wallace, G. (2004). Irresponsible ecotourism. Anthropology Today, 20(3), 1-2. Doi: https://doi.org/10.1111/j.0268-540X.2004.00265.x

Sandro, A. (1998). Introducción al turismo. Madrid: OMT.

Sauma, P. (2011). Las desigualdades intrarregionales y la reducción de la pobreza en la región Chorotega. Ciencias Económicas, 29(2), 331-397. Recuperado de https://docs.google. com/viewerng/viewer?url=https://revistas.ucr.ac.cr/index.php/economicas/article/ viewFile/7027/6712

Segura, G. (1998). Comentarios acerca de la campaña de promoción turística de Costa Rica en Estados Unidos, 1996-1997. San José, Costa Rica: Incae. Recuperado de http://www.incae. edu/ES/clacds/publicaciones/pdf/cen652.pdf

Tilly, C. (2000). La desigualdad persistente. Buenos Aires: Ediciones Manantial.

Universidad de Costa Rica. (s. f.a). Bachillerato en turismo ecológico. Recuperado de http://www. so.ucr.ac.cr/bachillerato-en-turismo-ecologico

Universidad de Costa Rica. (s. f.b). Plan de estudios bachillerato en turismo ecológico. Recuperado de http://www.so.ucr.ac.cr/sites/ucrso1/files/carreras/PLAN\%20DE\%20ESTUDIOS.pdf

Universidad Nacional.(s.f.a).Bachillerato en gestión empresarial del turismo sostenible. Recuperado de http://www.documentos.una.ac.cr/bitstream/handle/unadocs/2797/85.pdf?sequence

Universidad Nacional. (s. f.b). Gestión empresarial del turismo sostenible (Bachillerato). Recuperado de http://www.una.ac.cr/index.php/m-oferta-academica/gestion-empresarial-del-turismosostenible-bachillerato 